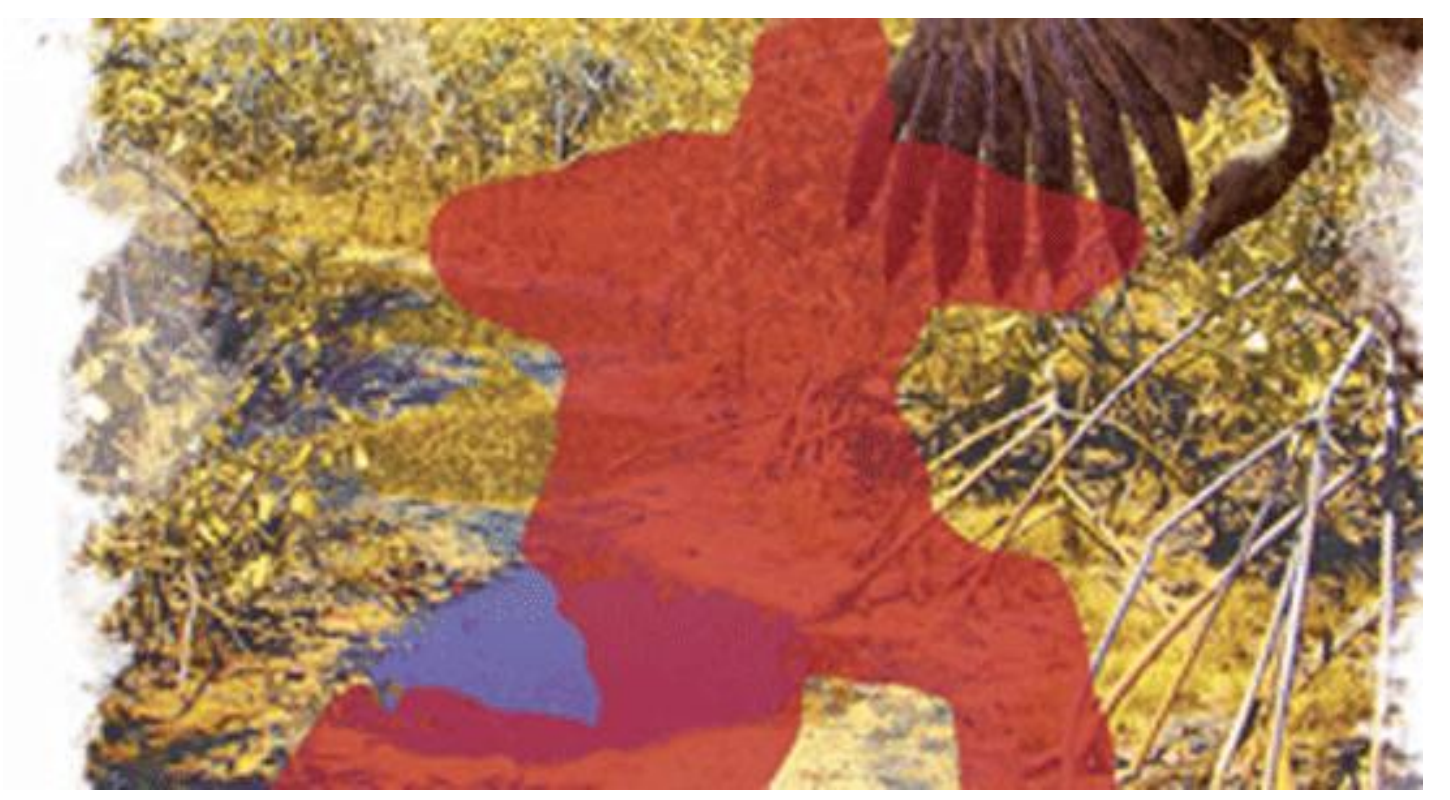

\title{
Los proyectos comunitarios como entornos de aprendizaje para la sostenibilidad en la formación inicial de maestros
}

(Community projects as learning environments for Sustainability in initial teachers' education)

\begin{abstract}
Lidia Ochoa Cañigueral ${ }^{1}$ Anna Maria Geli de Ciurana ${ }^{2}$

Resumen

En este artículo explicamos el papel que desempeña el proyecto comunitario de educación científica "Girona, ciudad semilla de ciencia" en la formación inicial de maestros. Se exponen reflexiones sobre la relación entre la educación científica y la educación para la sostenibilidad. A partir de la investigación acción y de la película Narradores de Javé se argumenta la importancia de abrazar la diversidad de puntos de vista para crear y potenciar entornos de aprendizaje adecuados.

Palabras clave: formación de maestros; educación para la sostenibilidad; Proyecto Polen; investigaciónacción; diversidad.

Abstract

This article explains the role of the science education community project "Girona, seed city for science" in University of Girona initial Teachers' training degree. We present reflections on the relationship between science education and education for sustainability that support the importance of creating and fostering appropriate learning environments. These reflections arise from the action research being carried out and the viewing of the film Narradores de Javé (the Storytellers, Brazil 2003).

Keywords: teachers formation; education for; Polen Project; investigation-action; diversity.

\footnotetext{
${ }^{1}$ Investigadora del Grupo de Investigación en Educación Científica y Ambiental (GRECA) - Universidad de Girona, España. Facultad de Educación y Psicología, Plaza Sant Domènec, 9, 17071-GIRONA (España) Contacto: lidia.ochoa@udg.edu

${ }^{2}$ Catedrática y Directora del Grupo de Investigación en Educación Científica y Ambiental (GRECA) Universidad de Girona, España. Facultad de Educación y Psicología, Plaza Sant Domènec, 9, 17071GIRONA (España). Contacto: am.geli@udg.edu
} 


\section{Una experiencia explicada a través de múltiples voces: todos somos narradores y protagonistas}

En el film Brasileño Narradores de Javé (2003), la población analfabeta de la pequeña ciudad de Javé encomienda a Antonio Biá la misión de escribir la historia de la ciudad, en un intento por detener la construcción de una represa hidroeléctrica que amenaza con destruir la aldea. A partir de los encuentros y entrevistas con Antonio, los diferentes personajes del pueblo empiezan a recordar y narrar sus versiones sobre grandes personalidades y acontecimientos locales.

Sin embargo, Antonio constata la tremenda dificultad de escribir alguna cosa cierta sobre la historia de Javé a causa de las múltiples versiones contradictorias que va compilando. Éstas son fruto del deseo que tienen todos los habitantes del pueblo por enfatizar el pasado glorioso de sus antepasados, aún cuando eso signifique cambiar sustancialmente la historia original. La ausencia de registros históricos anteriores también dificulta la transmisión de los hechos ocurridos en el pasado y propicia las divergencias entre narrador y narrador.

Finalmente, Antonio acaba escribiendo la historia de Javé, pero en vez de hacerlo para salvar el pueblo de la inundación, lo hace para salvar la memoria y el recuerdo de la aldea. Escribe los sucesos más recientes, las últimas páginas de la historia de Javé. Y lo hace a partir de su propia experiencia y de las narraciones de sus vecinos.

La película, entre otras muchas cosas, nos muestra la importancia de la alfabetización y del registro escrito. También resalta las múltiples versiones que pueden existir de un mismo hecho, debido a las diferentes percepciones, deseos y vivencias personales de las personas. La historia no es sólo un listado cronológico de hechos. Es cómo se vivieron esos hechos y situaciones y cómo los interpretamos.

De un modo parecido, los métodos de investigación cualitativos nos permiten recoger datos que incluyen opiniones, percepciones y vivencias de las diferentes personas y colectivos implicados en un proyecto, suceso o proceso. El análisis de los registros de fuentes diversas y complementarias nos permite entender qué sucede y cómo lo perciben los diferentes actores.

El proyecto comunitario "Polen, Girona ciudad semilla de ciencia" tiene muchos significados, tantos cómo personas participantes. A partir de la investigación que se lleva a 
cabo recogemos e interpretamos las fortalezas y debilidades del mismo, y además contribuimos a escribir la historia del proyecto y de sus protagonistas. En este artículo nos centramos en los y las estudiantes de magisterio de la Universidad de Girona, una pieza esencial para mejorar la educación científica y ambiental en las etapas de educación infantil y primaria. La historia y significado del Proyecto los creamos entre todas las personas que participamos en él: todos somos narradores y protagonistas.

Una de las características principales de la Educación para la Sostenibilidad es el diálogo entre posiciones y visiones distintas, a veces contrarias pero siempre complementarias. Si queremos avanzar hacia la Sostenibilidad estamos condenados a entendernos, a buscar puntos comunes y consensos.

Esta visión compleja, fruto de compartir miradas, problemas y soluciones es la que convierte a los proyectos comunitarios en entornos de aprendizaje privilegiados. Un claro ejemplo es el proyecto "Olhares cotidianos da Certificação Turismo CO2 Neutro: logos e grafias de uma transformação na APA Itacaré /Serra Grande". 3 Para poder expresar y comprender las diferentes perspectivas a veces hacen falta algo más que palabras. El proyecto "Olhares Cotidianos..." utiliza la fotografía como instrumento para captar y expresar percepciones, anhelos y motivaciones. Existen múltiples y diversos lenguajes y la utilización de los mismos enriquece el diálogo y el entendimiento. En la película Narradores de Javé, en el proyecto "Girona, ciudad semilla de ciencia” y en la experiencia de "Olhares Cotidianos..." se utilizan las narraciones para expresar y comprender qué está pasando. A veces la narraciones son orales, otras escritas y algunas incluso visuales.

\section{Educación científica para la sostenibilidad}

Desde hace más de un cuarto de siglo se considera que la formación científica es un objetivo clave de la educación en el sentido que se relaciona estrechamente con la capacidad de pensar y actuar en un mundo en el que la ciencia y la tecnología influyen de manera muy importante en nuestras vidas (GIL;VÍLCHEZ, 2006).

\footnotetext{
${ }^{3}$ Proyecto financiado por la Fundação de Amparo à Pesquisa do Estado da Bahia (Fapesb) con la implicación de Movimento Mecenas da Vida, Universidade Estadual de Feira de Santan (Uefs), Universidades Estadual de Santa Cruz (Uesc-Brasil), Universidade Estadual de Campinas - Brasil (Unicamp) y UdG (Universitat de Girona - España).
} 
En los últimos años ha emergido un notable interés y auge de la ciencia escolar en las etapas más tempranas y una rápida expansión de los museos y centros de ciencia donde la consigna es "prohibido no tocar". Por otro lado y de manera generalizada van apareciendo nuevos objetivos curriculares y estándares nacionales que parecen desarrollarse paralelamente a un creciente interés internacional en evaluar y medir las competencias y aprendizajes de los alumnos en algunas áreas de conocimiento. La utilización de pruebas como PISA y TIMSS $^{4}$ son ejemplos ilustrativos en el ámbito de la educación científica.

Jenkins (2009) dedica todo un artículo a explorar y comentar una selección de informes y documentos oficiales de reformas en educación científica y llega a la conclusión que la gran mayoría, si no todos, acaban siendo declaraciones de intenciones o deseos en vez de reformas actuales/reales. El reto actual es materializar y concretar dichos informes en políticas y planes de acción que promuevan e impulsen una mejora real en las clases y escuelas. En este sentido la formación de docentes adquiere un rol crítico esencial.

Estos cambios se suceden al mismo tiempo que confluyen otras tendencias a nivel nacional e internacional. Son ejemplos la creación del Espacio Europeo de Educación Superior (EEES) y la reorientación de los currículos hacia una perspectiva competencial. El conocimiento factual es imprescindible pero por sí solo no es suficiente para afrontar los retos del siglo XXI. Es necesario tener conocimientos y capacidades y saber cuándo movilizarlos y de qué manera hacerlo. A partir de esta idea surge el enfoque de la educación basado en competencias.

Una competencia requiere de la combinación de capacidades cognitivas y prácticas que se interrelacionan, conocimiento (incluido el conocimiento tácito), motivación, valores, actitudes, emociones y otros componentes sociales y de comportamiento que pueden ser movilizados para una acción efectiva en un contexto y situación determinados (OECD, 2002).

Según el marco evaluativo de PISA (OECD, 2006) la competencia científica hace referencia a los conocimientos científicos de un individuo y al uso de ese conocimiento para identificar problemas, adquirir nuevos conocimientos, explicar fenómenos científicos

\footnotetext{
${ }^{4}$ PISA es el acrónimo de "Programme for International Student Assessment", y TIMSS de "Trends in International Mathematics and Science Study".
} 
y extraer conclusiones basadas en pruebas sobre cuestiones relacionadas con la ciencia. Asimismo, comporta la comprensión de los rasgos característicos de la ciencia, entendida como un método del conocimiento y la investigación humanas, la percepción del modo en que la ciencia y la tecnología conforman nuestro entorno material, intelectual y cultural, y la disposición a implicarse en asuntos relacionados con la ciencia y con las ideas de la ciencia como un ciudadano reflexivo. En este contexto, la competencia científica se define como la capacidad de utilizar el conocimiento y los procesos científicos, no solo para comprender el mundo natural, sino también para intervenir en la toma de decisiones que lo afectan.

El proyecto que nos ocupa está enfocado principalmente a las etapas de educación infantil y primaria y desde su inicio se tuvo presente que hoy en día la educación científica (es decir, la educación que tiene como principal objetivo mejorar y desarrollar la competencia científica) se debe situar en el marco de la educación para la sostenibilidad.

El enfoque de la Educación para la Sostenibilidad contribuye a la educación científica aportando algunos de sus puntos clave como los que Tilbury definió en 2005: la búsqueda de la relevancia, la perspectiva holística y critica, la orientación en valores, la orientación a la acción y la incorporación de una dimensión de futuros. Además, esta se caracteriza por incorporar situación en el paradigma de la complejidad, perspectiva holística de la realidad y también del estudiante como individuo y parte del colectivo, metodología participativa, establecimiento de espacios de reflexión y democracia, clarificación de valores para la sostenibilidad y contextualización local-global (JUNYENT; GELI; ARBAT, 2003).

Las Naciones Unidas declararon la Década de la Educación para el Desarrollo Sostenible (2004-2015), basándose en la visión de un mundo donde todos tengan la oportunidad de recibir una educación y aprender valores, comportamientos y modos de vida necesarios para el advenimiento de un futuro sostenible y una transformación positiva de la sociedad.

Existe un consenso mundial sobre que el avance hacia el desarrollo sostenible depende de las acciones de los ciudadanos en todos los aspectos de la vida, lo que supone adoptar cambios en su manera de pensar, vivir y actuar. Partiendo de esta consideración 
también podemos decir que se está de acuerdo en la necesidad de cambios en la sociedad, para los cuales deben establecerse marcos de actuación que faciliten la participación, la concienciación, la educación y la capacitación. (International Union for Conservation of Nature - IUCN 2005). Todo ello requiere proporcionar oportunidades promover la perspectiva de la sostenibilidad mediante todas las formas de educación y formación.

\section{Proyecto "Girona, ciudad semilla de ciencia"}

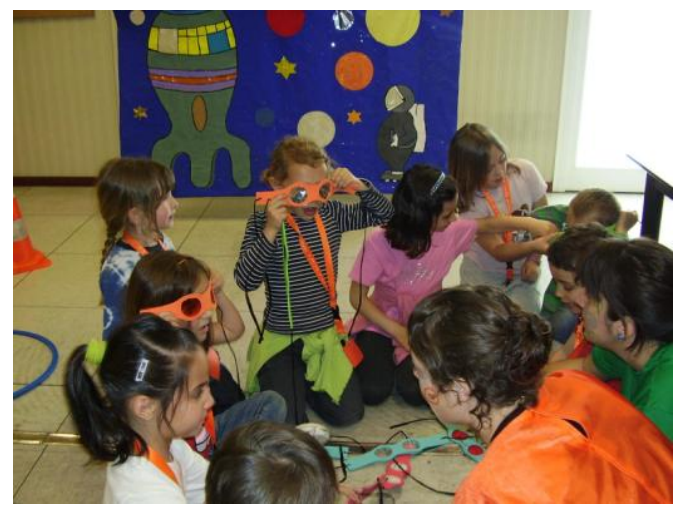

“Girona, ciudad semilla de ciencia” (2009)

El proyecto comunitario de educación científica "Girona, ciudad semilla de ciencia" empezó como parte del proyecto Europeo "Pollen, seed cities for science: a community approach for a sustainable growth of science education in Europe" (www.polleneuropa.net). El Informe Rocard (2007) lo destaco como una de las experiencias ejemplares en educación científica en Europa.

Polen es un proyecto europeo de investigación y desarrollo financiado por el Sexto Programa Marco de la Comisión Europea que se llevó a cabo entre los años 2006 y el 2009. Doce países participaron como socios y durante los tres años de duración del proyecto se desarrollaron doce iniciativas paralelas: una ciudad semilla de ciencia en cada país. La ciudad semilla de ciencia en España fue Girona. El principal objetivo del proyecto era la promoción e impulso a la educación científica en las etapas de educación infantil y 
primaria (niños y niñas de 3 a 12 años) con un enfoque basado en la investigación ${ }^{5}$ y experimentación y desde una aproximación comunitaria.

Definimos la metodología didáctica de investigación escolar como el desarrollo de estrategias de conocimiento, basadas en la investigación personal - y colectiva - del alumno, que requieren: la concreción del problema a investigar, explicitar las ideas previas, la elaboración y selección de hipótesis de trabajo, el diseño y aplicación de instrumentos de investigación y la elaboración y comunicación de las conclusiones (DEL CARMEN, 1988, p.12).

El proyecto "Girona, ciudad semilla de ciencia" lo coordina el Grupo de Investigación en Educación Científica y Ambiental (GRECA-UdG) y lo impulsan tres instituciones locales: Ayuntamiento de Girona, Departamento de Educación de la Generalitat de Cataluña (gobierno autonómico) y la propia Universidad de Girona ${ }^{6}$ El proyecto comunitario se centra en dos ejes prioritarios de acción: por un lado en el asesoramiento y formación permanente de maestros y maestras de las etapas de educación infantil y primaria. Desde el curso 2007-08, y en el marco de este proyecto, se ha trabajado principalmente con 6 centros de educación infantil y primaria de la ciudad de Girona, con la finalidad de promover la educación científica basada en la experimentación e indagación. Principalmente, se han realizado actuaciones de asesoramiento y apoyo a los proyectos de educación en ciencias de los centros escolares que decidieron participar en Polen. Por otro lado, también se organizan y realizan actividades y jornadas que implican la participación de la comunidad educativa de la ciudad y su ámbito de influencia. El ejemplo más emblemático es la Feria de la Ciencia dirigida principalmente a un público infantil (niños y niñas de 3 a 12 años).

La Feria de la Ciencia para Pequeños Grandes Científicos y Científicas es una actividad de divulgación y de educación científica que se ideó con el objetivo de crear una jornada lúdico-educativa donde pudieran participar los grupos clase que participan en el proyecto a lo largo del curso escolar. Con el paso del tiempo se ha consolidado como un espacio comunitario donde se promueve la colaboración entre los diversos agentes que

\footnotetext{
${ }^{5}$ En inglés Inquiry Based Science Education (IBSE).

${ }^{6}$ Durante el periodo financiado por el proyecto Europeo (2006-2009) también se contó con la coordinación de la empresa Pau Education.
} 
forman la comunidad educativa y científica de la ciudad de Girona y área de influencia. En las tres ediciones realizadas han participado docentes y alumnos de infantil, primaria, secundaria y estudios universitarios, instituciones locales y empresas privadas relacionadas con la educación y divulgación científica.

Desde el grupo de investigación GRECA y el área de didáctica de ciencias experimentales de la Universidad de Girona se aposto firmemente en que la Feria era también una magnífica oportunidad para involucrar y hacer partícipes a los estudiantes de magisterio en educación infantil y primaria.

\section{Participación de los y las estudiantes de magisterio}

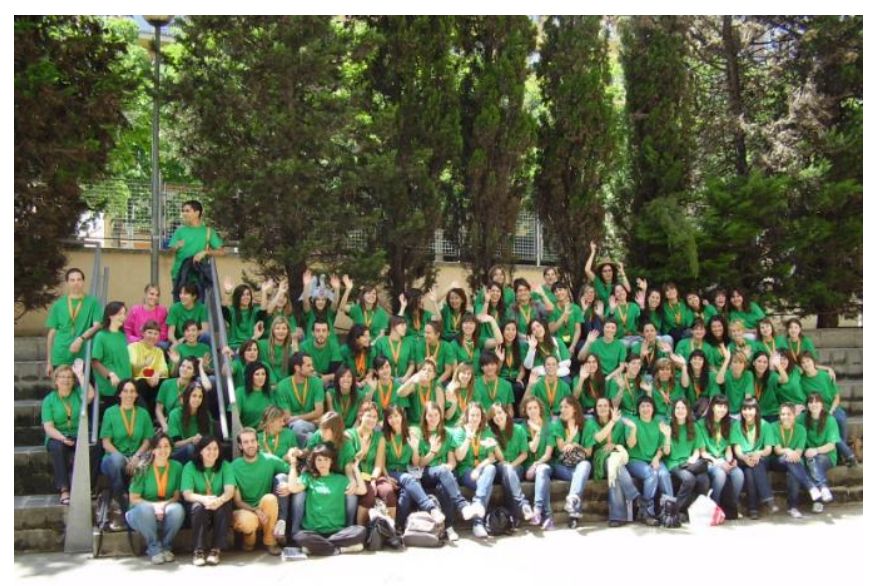

Estudiantes de magisterio (2009)

Los estudiantes de magisterio participan en el proyecto comunitario básicamente de dos maneras diferentes: desarrollando un taller de ciencia como parte del trabajo práctico en el marco de las asignaturas de didáctica de las ciencias y conocimiento del medio natural o bien como voluntarios de segundo o tercer curso, realizando tareas de apoyo a la organización o involucrándose en actividades concretas.

Hasta el curso académico 2006-07, el trabajo practico de las asignaturas de didáctica de las ciencias consistía en que el alumnado diseñaba y exponía sus propuestas didácticas elaboradas al docente y al resto de los compañeros de clase. Durante el curso 2007-08 se modificaron los trabajos prácticos de tal manera que las propuestas didácticas diseñadas y elaboradas por los alumnos se aplicaran en un Taller de Ciencia real con la participación de niños y niñas, dentro de las actividades programadas en la Feria de la 
Ciencia. El Taller de Ciencia se dirige a niños y niñas de educación infantil y primaria de centros escolares que participan en el proyecto Polen, "Girona, ciudad semilla de ciencia". Se trata de una propuesta didáctica basada en la observación, indagación y experimentación donde los niños y niñas son los protagonistas.

Los objetivos del Taller de Ciencia, desde el punto de vista de los y las estudiantes de magisterio, son:

- Aproximarse a la realidad docente en ciencias experimentales conectando el contexto teórico con la práctica (movilización de capacidades para desarrollar competencias profesionales);

- Establecer vínculos de trabajo en ciencias entre los alumnos de magisterio con los centros escolares;

- Reflexionar sobre la práctica docente para analizarla y mejorarla.

Los estudiantes de Magisterio preparan y realizan los talleres en el marco de la Feria de la Ciencia, posteriormente reflexionan sobre su práctica docente, su implicación en la comunidad escolar y evalúan el desarrollo del taller.

\section{Como voluntarios}

A raíz del entusiasmo manifestado por muchos estudiantes, a partir del curso 20082009 se ofreció la posibilidad de participar en el proyecto Polen de forma voluntaria a los y las estudiantes de segundo curso, es decir a los que el curso anterior participaron en la primera edición de la Feria de la Ciencia. Se convocó una reunión con todas las personas interesadas, explicó detalladamente en qué consiste el proyecto Polen y se apuntaron diferentes vías de colaboración, dejando siempre la posibilidad de encontrar otras nuevas que se adaptaran a los intereses y motivaciones de los colaboradores.

En sucesivas reuniones, de periodicidad variable pero cercana a una reunión mensual o bimensual, se fueron definiendo tareas y compromisos. Finalmente se establecieron diferentes tipologías de colaboración:

- Acompañamiento de las sesiones de formación dirigidas a maestros - Las y los estudiantes voluntarios asisten siempre que quieren a las sesiones de formación de 
los maestros, tanto en las conjuntas (maestros de todas las escuelas implicadas en el proyecto) como las realizadas en las propias escuelas.

- Apadrinamiento de grupos (un estudiante de $2^{\circ}$ o $3^{\circ}$ apoya a un grupo de $1^{\circ}$ para la realización de su taller) - Esta práctica tiene presentes dos objetivos simultáneos. En primer lugar, ayudar y guiar en el proceso de elaboración del taller a un grupo de primero. En segundo lugar, fomenta las habilidades docentes del estudiante de $2^{\circ} \mathrm{o}$ $3^{\circ}$ que realiza la tarea de apadrinar, ya que debe orientar el trabajo realizado por el grupo de $1^{\circ}$ y aconsejar desde la experiencia del año anterior.

- Organización de talleres adicionales - Estos talleres están pensados para ser utilizados durante los tiempos de espera de los grupos que asisten a la Feria, ya sea al llegar o entre taller y taller. Los estudiantes voluntarios se implicaron en el proceso de preparación de estos talleres y en su realización.

- Organización general de la Feria de la Ciencia - Los alumnos voluntarios forman parte de la organización general, haciendo múltiples tareas: preparar y entregar las acreditaciones, preparar los espacios y materiales, apoyar a los grupos de de estudiantes de primer etc.

- Supervisión de grupos y gestión - de los grupos durante la Feria de la Ciencia: los alumnos y alumnas hacían acompañamientos de los grupos de niños y niñas durante los tiempos de espera, información y logística etc.

Una vez realizada cada edición de la Feria de la Ciencia para Pequeños Grandes Científicos, se evalúa su resultado a diferentes niveles. Se pasan cuestionarios a maestros y alumnos de los estudios de magisterio, se recogen y analizan las reflexiones incluidas en los trabajos prácticos de los alumnos de primer y se realizan reuniones de evaluación (con colaboradores y miembros de las Instituciones implicadas en el proyecto). El análisis de las distintas valoraciones forma parte del estudio y monitoreo del proyecto "Girona..." que exponemos a continuación.

\section{Seguimiento y evaluación del proyecto: investigación acción y práctica reflexiva}

El proyecto de investigación que se está realizando tiene como objetivo realizar un seguimiento sistemático y una evaluación formativa del proceso de implementación y desarrollo del proyecto "Girona, ciudad semilla de ciencia”. Compartimos la perspectiva de 
Mateu y Vidal (1997) según la cual la investigación evaluativa es un conjunto de procesos para la obtención y análisis de información en el cual se puedan dar juicios de valor sobre el objeto, fenómeno o proceso investigado, como parte de la toma de decisiones sobre el propio proceso.

Se trata de una investigación evaluativa con un enfoque de investigación acción. Ésta puede ayudarnos a aprender a participar y cambiar nuestras prácticas de manera que se adapten a nuestra configuración personal mediante la creación de condiciones para el cambio en tres niveles - personal, profesional y político (Noffke 1997). Compartimos la idea que una forma adecuada de desarrollo profesional, como la Educación para la Sostenibilidad en sí misma, ha de ser contextualizada, y respetuosa hacia la creación de conocimientos de los que están involucrados en el proceso.

Parte de la investigación que se está llevando a cabo está orientada a elaborar una propuesta de mejora de la formación inicial de maestros y maestras. Es en este ámbito en el cual incluimos las reflexiones sobre el papel que pueden desempeñar los proyectos educativos como entornos de aprendizaje en la formación universitaria de los futuros maestros.

Los métodos utilizados para la obtención de datos son principalmente cualitativos. Realizamos entrevistas semi estructuradas, individuales y colectivas, a maestros en formación (estudiantes voluntarios) y en activo (representantes de cada escuela participante). Complementamos la recogida de datos y su triangulación con revisión documental de los materiales didácticos utilizados y con observación (participante y no participante). A partir del curso 2008-2009 también se diseñaron y utilizaron cuestionarios para conocer las motivaciones, intereses y percepciones de los estudiantes que participaron voluntariamente en el equipo del proyecto.

\section{Resultados}

En cuanto a los objetivos de las asignaturas de didáctica de las ciencias, se han conseguido importantes progresos en relación a motivar, a los futuros maestros, y a desarrollar interés en la enseñanza de las ciencias experimentales y el conocimiento del medio natural, han sido plenamente conseguidos. Los estudiantes manifestaron de manera 
explícita que durante el proceso su motivación había ido en aumento y se encontraron mucho más receptivos a los planteamientos de las ciencias naturales y a las estrategias metodológicas para su enseñanza. En los cuestionarios post-taller casi la totalidad de alumnos afirmaron que el interés y motivación para preparar y realizar propuestas didácticas basadas en la experimentación había aumentado debido a la experiencia de participación en la Feria de la Ciencia.

Un elemento que destacan es el apropiarse y vivenciar de manera real un proyecto educativo, lo que - utilizando sus propias palabras - les ha fortalecido su compromiso y responsabilidad educativa para llevarlo a cabo y aplicarlo con un sentido de calidad de enseñanza (OCHOA, 2009).

Del mismo modo se puede afirmar que la intervención educativa llevada a cabo ha favorecido el aprendizaje de cómo reflexionar sobre la práctica docente en ciencias. En las reflexiones grupales de los estudiantes destaca claramente el valor añadido que supone para los futuros y futuras maestras poder poner en práctica un diseño didáctico propio, aún más cuando la práctica ha sido repetida con diferentes grupos clase. Este factor ha permitido reflexionar sobre el trabajo y la práctica realizados y pulir los talleres utilizando la evaluación cómo herramienta de mejora.

\section{Voluntariado}

En un inicio fue difícil definir las colaboraciones y establecer niveles de compromiso adecuados a las necesidades e intereses. Durante las primeras reuniones parecía que no se avanzaba demasiado y los propios estudiantes pedían más implicación. Poco a poco el grupo fue cogiendo forma y finalmente la participación ha sido total, no sólo en la ejecución, sino en la fase de planteamiento de las dos últimas ediciones de la Feria.

Este hecho permite contar con más recursos humanos para desarrollarla, y una distribución de tareas que contribuye a la creación de un equipo de personas que se autogestiona y ofrece un apoyo que se ha convertido en un puntal de la organización. El equipo de voluntarios y voluntarias ha ido ganando autonomía e iniciativa a medida que ha avanzado el curso académico, además de generar un sentimiento de pertenencia y de comunidad de aprendizaje fuera de las clases ordinarias. 
Según las propias reflexiones de los estudiantes voluntarios, los y las voluntarias de segundo curso y tercer curso se han planteado tres aspectos básicos a la hora de decidir participar. En primer lugar destaca la propia voluntad de querer hacer más cosas en función de la experiencia del año anterior. En segundo lugar, porque la Feria de la Ciencia en concreto y el proyecto Polen en general constituyen un marco muy adecuado para introducir la participación de diferentes grupos y personas en un proyecto local y comunitario. Finalmente, en tercer lugar la participación se ha visto motivada para poder adquirir más experiencia en contacto con los niños y niñas, y tener un complemento práctico a la formación.

En cuanto a la participación en las sesiones de formación de maestros, los estudiantes que han asistido, lo han hecho como un maestro más para poder trabajar en equipo, con maestros en activo, poniéndose en situación real de lo que experimentan los niños en relación a los talleres que desarrollan, y adquieren herramientas para poder desarrollar más correctamente sus capacidades docentes en un futuro. Ha sido muy bien valorado por todos los que han asistido y han manifestado el interés por participar en el próximo curso.

En términos generales, los estudiantes destacan que las tareas desarrolladas que no han supuesto una dedicación excesiva en cuanto a tiempo, pero sí ha supuesto una recompensa sensacional en forma de aprendizaje. Este aprendizaje, los participantes voluntarios lo traducen de diversas formas. En primer lugar, el hecho de participar en este proyecto les permite tomar ideas y adquirir recursos para encarar su futuro profesional. Asimismo, el Proyecto Polen ha permitido que adquieran unos hábitos y unas pautas en el planteamiento, preparación, ejecución y valoración o evaluación de actividades dirigidas a niños y niñas.

Un elemento a destacar es que la carrera universitaria de Magisterio (antes de la entrada en vigor de los nuevos títulos de grado) no contempla las prácticas en contextos reales dentro de las asignaturas ordinarias. Las oportunidades que tienen sus estudiantes estar en contacto con los niños y poner en práctica sus habilidades es prácticamente nula (exceptuando el periodo de prácticas en la escuela, en el último curso de la carrera), es por 
eso que todos destacan la gran importancia de poder disfrutar de espacios como estos para poder hacer un acercamiento verosímil a su futura profesión.

Durante el curso 2008-2009 participaron 10 voluntarios, en el siguiente curso fueron más de 30 y no dejan de aumentar. Esta tendencia constituye un indicador claro del éxito de la iniciativa, y lo es aun mas porque los estudiantes no reciben nada a cambio de su participación, nada material o académico, se entiende, ya que ellos hacen que la experiencia les aporte muchísimas otras cosas, como las que ya hemos descrito en este escrito.

\section{Reflexiones finales}

A partir de los argumentos expuestos y de los resultados obtenidos a lo largo del proceso de investigación-acción, podemos afirmar que el proyecto comunitario objeto de estudio tiene un gran potencial como entorno de aprendizaje que complementa la formación inicial de maestros y maestras. Este potencial se basa en una de sus características esenciales: se trata de un proyecto y un contexto real, adaptado y desarrollado en base a las necesidades de la comunidad que participa. Cada persona o grupo implicado contribuye en el propio proceso de desarrollo del proyecto y en el aprendizaje colectivo que se va creando conjuntamente. Los estudiantes de magisterio aportan una dosis tremenda de energía e ilusión y reciben experiencia en la práctica profesional y vivencias personales muy intensas. El trabajo cooperativo y en red es un tema recurrente en la formación de maestros pero en raras ocasiones se brinda la oportunidad a los y las estudiantes de formar parte de un ejemplo concreto, real y que funcione. Esta es la riqueza del proyecto "Girona, ciudad semilla de ciencia".

Sin duda, el hecho de ir añadiendo agentes y colectivos en el proyecto supone una gran contribución, pero no hemos de olvidar que a medida que aumenta la complejidad también aumentan los retos asociados a ella. Se trata de un proceso de aprendizaje colectivo y la única manera de afrontar los retos y aprovechar las oportunidades es seguir aprendiendo juntos.

\section{Referencias bibliográficas}

DEL CARMEN, Luís M. (1988): Investigación del Medi i Aprenentatge. Barcelona, Graó. 
- (2006): Las actividades prácticas en contextos multiculturales de la educación infantil y primaria en Alambique, n.47, pp.56-64.

GELI, Anna. Maria., (ed.) (2006): Ambientalización curricular en l'ensenyament obligatori: una proposta de definició, caracterització $i$ estratègies. Monografies universitàries; 7. Barcelona, Departament de Medi Ambient i Habitatge.

GIL PÉREZ, Daniel; VILCHES, Amparo (2006). Como puede contribuir el proyecto PISA a la mejora de la enseñanza de las ciencias (y de otras aéreas de conocimiento)? Revista de Educación, extraordinario 2006, pp. 295-311.

JENKINS, Edgar W. (2009) Reforming school science education: a commentary on selected reports and policy documents, Studies in Science Education, 45: 1, 65 - 92.

JUNYENT, Mercè; GELI, Anna M.; ARBAT, Eva. (ed.) (2003). Ambientalización Curricular de los Estudios Superiores. Proceso de Caracterización de la Ambientalización Curricular de los Estudios Universitarios. Girona: Universitat de Girona - Red ACES.

JUNYENT, Mercè.; OCHOA, Lidia. (2006): Competència ambiental” en en Geli, A.M ; Pèlach, I (ed.) (2006) Aproximación a les competències en els nous títols de mestre. Colección Diversitas; 56. Girona. Universitat de Girona.

OECD (2002): Definition and Selection of Competences (DeSeCo): Theoretical and Conceptual Foundation. Strategy Paper JT00132752.

OECD (2006) PISA 2006. Marco de la evaluación. Conocimientos y habilidades en ciencias, matemáticas y lectura. Madrid. Ed. Santillana - MEC.

OCHOA, Lidia Cañigueral; FRIGOLA, Climent Darder; BERTRAN, Àdam Martínez; HERAS, Raquel Colas (2009). La Participación dels i de les estudiants en l'organitzación de la 2a edición de la Fira de la Ciència: una reflexión centrada en la tutoritzación i el voluntariat. Congreso Internacional UNIVEST'09. Girona: Universitat. [Consulta 22 gener 2010]. Disponible en: http://hdl.handle.net/10256/1836 
POLLEN PROJECT: Seed Cities for Science. Financiado por el Sexto Programa Marco de la Comisión Europea. Disponible en http://pollen-europa.net.

ROCARD, M. (dir.) (2007): Science Education Now: A Renewed Pedagogy for the Future of Europe, European Comission. Disponible en http://ec.europa.eu/research/sciencesociety/document library/pdf 06/report-rocard-on-science-education en.pdf.

TILBURY, Daniella (1995) Environmental education for sustainability: defining the new focus of environmental education in the 1990s, Environmental Education Research, 1, 2, 1995-214. 


\section{Revista do Laboratório de}

Estudos Urbanos do Núcleo

de Desenvolvimento da Criatividade

Para citar essa obra:

CAÑIGUERAL, Lidia Ochoa; CIURANA, Anna Maria Geli de. Los proyectos comunitarios como entornos de aprendizaje para la sostenibilidad en la formación inicial de maestros. RUA [online]. 2011, no. 17. Volume 1 - ISSN 1413-2109

Consultada no Portal Labeurb - Revista do Laboratório de Estudos Urbanos do Núcleo de Desenvolvimento da Criatividade http://www.labeurb.unicamp.br/rua/

Laboratório de Estudos Urbanos - LABEURB

Núcleo de Desenvolvimento da Criatividade - NUDECRI

Universidade Estadual de Campinas - UnICAMP

http://www.labeurb.unicamp.br/

Endereço:

Rua Caio Graco Prado, 70

Cidade Universitária "Zeferino Vaz" - Barão Geraldo

13083-892 - Campinas-SP - Brasil

Telefone/Fax: (+55 19) 3521-7900

Contato: http://www.labeurb.unicamp.br/contato 